\title{
AIMING FOR THE STARS
}

\author{
THERE IS STILL A HUGE AMOUNT WE DO NOT KNOW ABOUT THE \\ GALAXY WE INHABIT. THE MAJORITY OF THE MILKY WAY REMAINS \\ UNMAPPED AND MYSTERIOUS. PROFESSOR TOM BANIA OF BOSTON \\ UNIVERSITY IS CHIPPING AWAY AT THESE UNKNOWNS, USING THE \\ LATEST TELESCOPE TECHNOLOGY TO REVEAL EXACTLY WHAT IS FAR \\ BEYOND OUR PLANET'S NEIGHBOURHOOD
}

\section{TALK LIKE AN ASTRONOMER}

\section{CELESTIAL BODY}

Any natural astronomical object, such as stars, planets and asteroids.

\section{GALAXY}

A system comprising stars, gas and dust, held together by gravity.

\section{IONISED}

Atoms or molecules that carry an electrical charge.

\section{KINEMATIC DISTANCE}

Working out distances between celestial bodies by calculating their relative velocities.

\section{NEBULA}

A cloud of dust or gas in space.

\section{RADIATION}

Emission of energy as electromagnetic waves. The electromagnetic spectrum includes radio waves, microwaves, infrared, visible light, ultraviolet, $\mathrm{X}$-ray and gamma rays.

\section{SUPERNOVA}

A star that is undergoing an explosion, resulting in the scattering of its mass and a sudden increase in brightness.
With every passing year, leaps forward in technology, scientific equipment, computing power and innovative thinking have paved the way for ever-greater discoveries in astronomy. Professor Tom Bania, of Boston University's Department of Astronomy, is using the latest telescopes and galactic mapping expertise to uncover the locations of nebulae within the Milky Way.

HII REGIONS: BUBBLES OF SPACE GAS The most massive stars in our galaxy, known as $O B$ stars, are 20-50 times the mass of our Sun. They emit a huge amount of ultraviolet radiation over the course of their (comparatively short) lifetimes, which cause hot bubbles of ionised gas to form. These gas bubbles, a type of nebula, are known as HII regions, and are where Tom's research currently focuses.

An OB star has an immensely powerful nuclear reactor at its core, which fuses together atomically light elements (such as hydrogen and helium) into heavier elements. When it reaches the end of its life, a massive $O B$ star becomes a supernova - in other words, explodes - and disperses these elements into space. Eventually, they may coalesce into planets and other celestial bodies. The existence of life depends on supernovae. "Every iron atom in our blood, for example, was formed deep inside an OB star," says Tom.

\section{NEW NEBULAE}

Tom's research team works on discovering previously unknown HII regions in our galaxy. "These new nebulae are extremely far away from the Sun," he says. "Previous generations of telescopes couldn't detect the signals they gave off. We also just didn't know where to look - space is a big place, after all."

Different telescopes detect electromagnetic radiation of different wavelengths. With visible light, for instance - the same wavelengths we use for vision - we can only see about one-third of the distance to the centre of the Galaxy. We can use infra-red and microwaves to see further, and radio waves to see the furthest of all - anywhere within the Galaxy, in fact. "The dust in the Galaxy absorbs electromagnetic radiation. Visible light is the most absorbed and radio light the least. This means that the extremely bright radio emission from $\mathrm{HIl}$ regions makes it possible to find these nebulae even if they are at vast distances from Earth," Tom explains. "Through discovering over a thousand distant HII regions, we can now drastically improve our map of the Milky Way."

\section{A BIG TELESCOPE FOR A BIG JOB}

"Modern telescopes mostly benefit from advances in electronics and computer power, and have come on a long way since I began observing HII regions in the 1970s," says Tom. 


\section{TOM'S TOP TIPS FOR STUDENTS}

1 To quote the mythologist Joseph Campbell, "Follow your bliss." I am a working-class kid, and I followed my bliss. Remember, as an undergraduate, you are the customer - you pay them for your education. As a graduate you become an employee, and effectively get paid to be a student. That's sweet!

2 Learn electronics and programming. These skills will help you get a job anywhere, not just in astronomy.

3 If you are not happy doing what you are doing,

3 find something else that does make you happy. Life is too short.
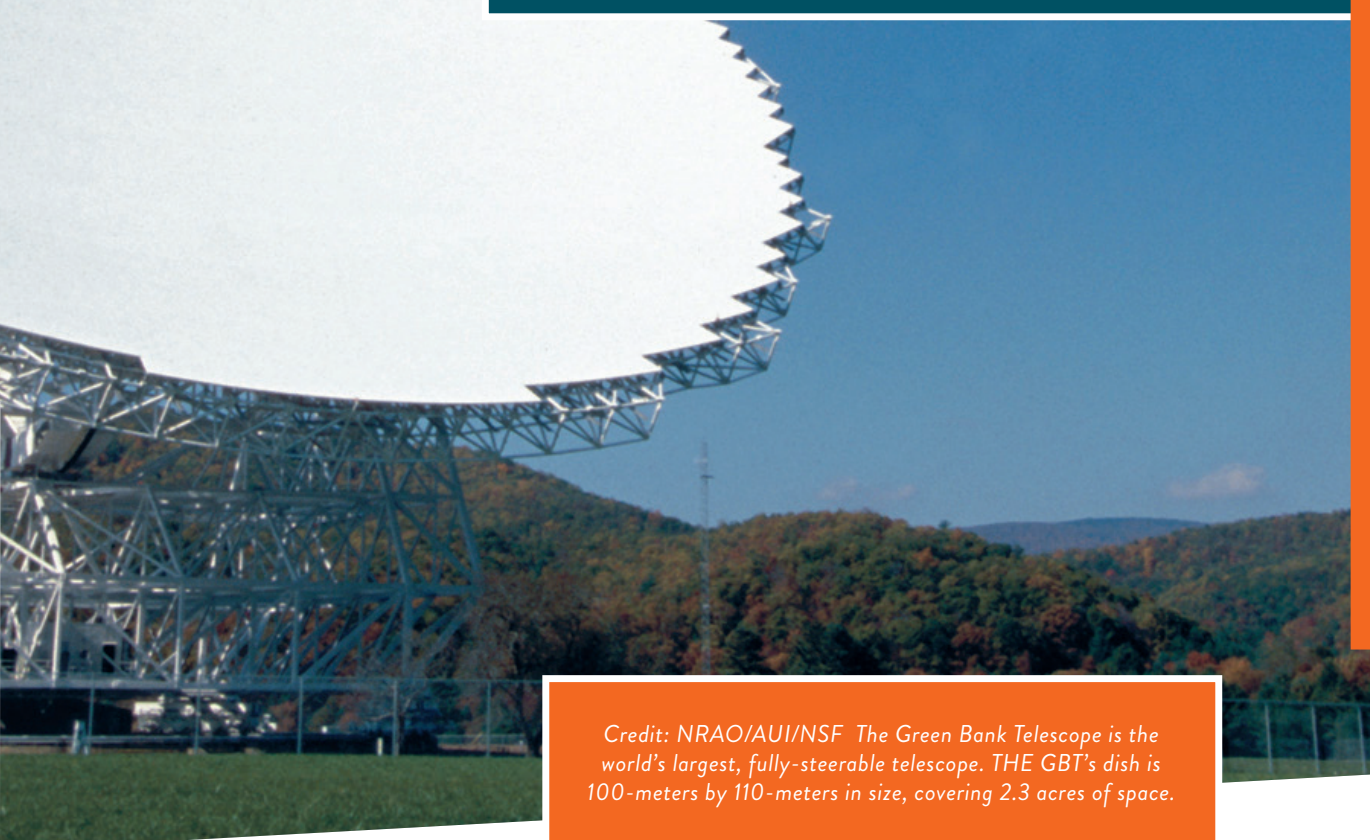

Credit: NRAO/AUI/NSF The Green Bank Telescope is the

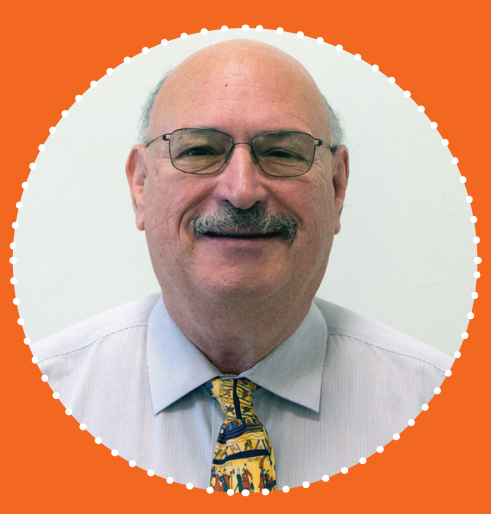

PROFESSOR TOM BANIA

Astronomy Department Institute for Astrophysical research, Department of Astronomy Boston University, USA

\section{FIELD OF RESEARCH}

Astronomy

$\ldots \ldots \ldots \ldots$

RESEARCH PROJECT

Detecting and mapping the presence of HII regions

throughout the Milky Way.
The Green Bank Telescope (GBT), which was crucial for his research, also benefits from clever mechanical design. It essentially looks like a TV satellite dish but is a whopping 100 metres in diameter. "The GBT is unique because it can pick up frequencies spanning a very wide portion of the radio wave spectrum," says Tom.

HII regions are highly ionised, and the charged particles within them are steadily combining to form neutral atoms. This process gives off a signal: when an electron drops down from a very high energy level, it produces a photon at a specific frequency. The GBT can be 'tuned' to detect this frequency, and so detect the presence of $\mathrm{HII}$ regions.

\section{BUILDING THE GALACTIC MAP}

Even once these signals are picked up, it's difficult to tell how far away the HII regions are. "Determining the distances to celestial bodies is one of the hardest parts of astronomy," says Tom. One clever technique astronomers use is known as "kinematic distance', which involves measuring how fast an object is moving relative to Earth. Tom explains, "The relative velocity of the HII region, with respect to the Earth, can be used to determine the distance to the nebula and this is known as a 'kinematic' distance. The word stems from a Greek root that means 'motion'." To measure this velocity, astronomers use the 'Doppler effect', where wave frequencies change due to the relative motion between the object and the measurer. It's the same effect that makes police sirens drop in pitch when the vehicle is moving away from you. Once they have determined this relative velocity, astronomers can derive the kinematic distances to HII regions using their knowledge of how the Galaxy rotates about its centre.

Tom's research team aims to produce 'the first complete map of where massive stars are forming in the entire Milky Way disc'. This is no small task. "We have just completed our Southern Hemisphere HII Region Discovery Survey," says Tom. "We found over 500 previously unknown HII regions, adding to a total of over 2,500 nebulae recorded in the Milky Way." Now, they are working to understand exactly how the Milky Way rotates, so that they can determine kinematic distances between $\mathrm{HII}$ regions and add these points to the galactic map.
HOW ANSWERS LEAD TO QUESTIONS

The project is also giving interesting insights into the wider galactic make-up. There are more stars (including more $O B$ stars) near the centre of the Galaxy, which means that you would expect there to be more heavy elements there - which is exactly what they found. Nonetheless, at any given distance from the centre, you would also expect there to be about the same abundance of heavy metals in any direction. The researchers have instead found localised pockets of heavy element abundance, and are currently trying to work out why this is the case. "This result would not have been possible without a large sample of HII regions," says Tom.

The work of Tom and his team highlights just how much there is more to learn. The sum knowledge of astronomy is growing exponentially as more and more observations become possible, which means that exciting discoveries are happening every year. As with $\mathrm{HIl}$ regions, each discovery also raises a raft of new questions, which will shed more light on what makes our universe tick. 


\section{ABOUT ASTRONOMY}

THE ART OF DISCOVERY

Since there is still plenty that we don't know about the universe, much of astronomy is about discovery. "I have had my share of discoveries, and they are extremely satisfying - more than satisfying, actually," says Tom. "Nothing worthy of the Nobel Prize, but each time I celebrate with colleagues, with fine wine and gourmet food."

Of course, as with any science, there are sometimes setbacks too. "I have a couple of missed discoveries, and these haunt me. One was on a figure in one of my first papers, an effect that just looked like 'noise' (that didn't appear to be a real signal), but thirty years later was found to be a spiral arm of the Milky Way."

\section{WHAT KEEPS ASTRONOMERS UP}

\section{AT NIGHT}

Like any field, astronomy has its fair share of challenges. "The practical challenges are the same as with any science," says Tom. "Accessing the latest instruments, getting funding, finding permanent jobs... Same old, same old."

Where astronomy becomes more unique is in the sheer scale of the undiscovered. "If our interpretations are valid, only about $4 \%$ of the universe is made up of atoms that we are familiar with," Tom continues. "The remainder is dark matter and dark energy, which we know very little about - and that's uncomfortable."

\section{THE NEXT GENERATION}

"There is great satisfaction in sharing mutual joy in the process of research with my students," says Tom. "I have learned that one cannot teach how to do research, only show by example. Half the time it doesn't take, but when it does, many go on to an astronomical research career. Then, I enjoy collaborating with them for many more years."

Boston University has weekly free open nights for the general public, where they are invited to look round the campus observatory. Green Bank Observatory also runs the 'Physics Inspiring the Next Generation' summer camps, where Tom often mentors or lectures. "These kids are awesome!" he says. "Excited, engaged the whole deal."

\section{HOW TO BECOME AN ASTRONOMER}

- According to UK Uni, the top UK universities for physics and astronomy are the Universities of St Andrews, Cambridge, Oxford and Durham.

- According to Top Universities, the top USA universities for physics and astronomy are MIT, Harvard, Stanford and California, Berkeley.

- In the UK, there are 'Space Schools' for teenagers based in the Universities of Leicester, Kent and Glasgow.

- In the US, there are similar projects to the Green Bank Observatory's summer camp in several states. Arizona has the Astronomy Camp (astronomycamp.org) which offers 'immersive' STEM camps for teenagers, including real scientists as mentors.

- In the USA, salaries for astronomers tend to fall between $\$ 111,663$ and $\$ 130,927$, according to salary.com.

\section{PATHWAY FROM SCHOOL TO ASTRONOMY}

Tom recommends high school students take maths, physics, and astronomy if it's available. Chemistry also helps, and he advises learning computer programming.
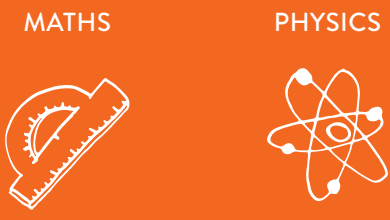

ASTRONOMY

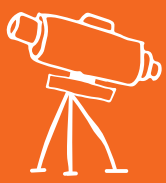

COMPUTER

PROGRAMMING

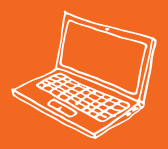

CHEMISTRY

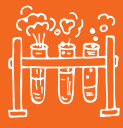

To become a fully-fledged astronomer, a degree and postgraduate qualification is usually required, and $\mathrm{PhDs}$ are common. Undergraduate subjects like astronomy, physics, maths, astrophysics, geophysics and space science can lead to a career as an astronomer. 


\section{0 \\ HOW DID PROFESSOR TOM BANIA BECOME AN ASTRONOMER?}

What led you to choose astronomy?

I started university as a chemistry major. In my junior year at Brown University, I discovered that I could actually get paid to do astronomy. That's what sealed the deal. I have had great scientific mentors too, especially my PhD advisor.

What were your interests as a child? Sports of all types were a big interest, as well as reading anything I could get my hands on. I always knew that I wanted to be a scientist. I was helped greatly by Sputnik (the first satellite ever launched by humankind launched by Russia), which kicked off the 'space race' between Russia and the USA. As a consequence, the USA funded a host of summer programmes for kids, intended to inspire the next generation of American scientists. It certainly worked for me!

\section{What has helped you become a successful} scientist?

Being smart and motivated, and being able to do things with my hands. I believe that the most important trait for a scientist is to have that 'fire in the belly'. I have seen many people much smarter than me fail as researchers, often because things had been too easy for them up to that point, and also because there is a big difference between following instructions and taking tests, compared to defining your own problems and tackling them through research.

How do you overcome obstacles in your research?

Stick with it. That's why it's called 'REsearch', after all. When really stuck, I find something else to do for a while, then try again. This can be years - I have examples where I tried to make observations that were technically impossible, but 25 years later, advances in scientific instruments have allowed me to make them.

What career goals do you have left to achieve?

None really - I am 70! 